\title{
EXTRACORPOREAL SHOCKWAVE TERAPY TO TREAT CHRONIC MUSCLE INJURY
}

\author{
Diego Costa Astur ${ }^{1}$, Bruno Santos ${ }^{1}$, Eduardo Ramalho de Moraes ${ }^{1}$, Gustavo Gonçalves Arliani ${ }^{1}$, \\ Paulo Roberto Dias dos Santos ${ }^{1}$, alberto de Castro Pochin ${ }^{1}$
}

\section{ABSTRACT}

Objective: To evaluate the low energy extracorporeal shock waves therapy (ESWT) associated with physical therapy in the treatment of chronic muscle injuries classified as grades 2 and 3 in the lower limbs of amateur athletes. Methods: Eight athletes presenting with lower limb muscle injury for more than three weeks were treated with physiotherapy and ESWT. We evaluated the following parameters during treatment: palpable gap, muscle strength, pain, and Tegner score, as well as ultrasound image features and the ability to return to sports practice. Results: The average time of the first evaluation of the injury was 8.75 weeks. All patients presented muscle strength grade $\mathrm{V}$ after eight weeks. The pain score evolved from 5.75 to 0.5 points of the visual analogue scale (VAS), at the end of the treatment. The Tegner score after treatment was six points on average. Patients returned to sports practice after 8.14 weeks. Conclusion: ESWT associated with physical therapy proved to be effective to treat long-term muscle injury, with good performance and the ability to return to sport practice for all patients. Level of Evidence IV, Case Series, Prospective Study.

Keywords: Wounds and injuries. Athletes. Lower extremity. Muscle, skeletal.

Citation: Astur DC, Santos B, Moraes ER, Arliani GG, Santos PRD, Pochini AC. Extracorporeal shockwave terapy to treat chronic muscle injury. Acta Ortop Bras. [online]. 2015;23(5):247-50. Available from URL: http://www.scielo.br/aob.

\section{INTRODUCTION}

Muscle injuries are common and considered the most prevalent musculoskeletal injury among nearly all sports categories. ${ }^{1,2}$ It corresponds to $46 \%$ of football injuries, ${ }^{3} 31 \%$ of soccer injuries, ${ }^{4}$ $17.7 \%$ in basketball, ${ }^{5}$ and $10.4 \%$ of injuries in rugby. ${ }^{6}$ Furthermore, thigh muscle injury is the most common injury in athletics. ${ }^{7}$ However, little progress has been made on studying muscle injuries in recent decades when compared to other pathologies related to sports. It is believed that the ideal way to define, classify and treat these injuries is not yet known. The lack of pattern in defining injuries makes them so different and difficult to compare with each other. Furthermore, the muscles are very different in size, shape, function and anatomical organization, which further complicates the existence of an ideal classification system. ${ }^{8,9}$

The muscles with proximal and distal insertion involving two joints (biarticular) are the most frequently injured. These are present in most of the muscles of lower limbs involved in sport gesture in sports such as football. For this reason, most muscle damage occurs in the four major muscle groups of the lower limbs: $37 \%$ in the knee flexor muscles, $23 \%$ in the thigh adductors, $19 \%$ in quadriceps muscle, and $13 \%$ in calf muscle. ${ }^{4}$ There is no consensus to distinguish acute injury from chronic injury. Histological features of chronic injury make that injury difficult to treat, probably due to formation of scar tissue that impairs the correct functioning of contracting and stretching the muscle fiber. Extracorporeal shock wave therapy (ESWT) is based on the formation of a single pulse acoustic wave generated by either an electromagnetic, electro-hydraulic, or piezoelectric source, able to increase the blood flow at the treatment site by inducing a healing process mediated by inflamation. ${ }^{10,11}$ However, there are no literature studies using this therapeutic modality for the treatment of muscle injuries.

The aim of the study was to evaluate the clinical results and return to sports activities obtained in a series of cases of longstanding muscle injuries grade II and grade III in lower limbs of athletes treated with low energy ESWT associated with physical therapy.

\section{METHODS}

This study was approved by the Medical Ethics Committee of this institution under protocol number 767337 and all patients signed a Free and Informed Consent form.

During the period from June 2013 to September 2014, eight patients (six men and two women) with muscle injury of the

All the authors declare that there is no potential conflict of interest referring to this article.

1. Universidade Federal de São Paulo, Escola Paulista de Medicina, Department of Orthopedics and Traumatology, São Paulo, SP, Brazil.

Work developed at Universidade Federal de São Paulo, Escola Paulista de Medicina, Department of Orthopedics and Traumatology, Sports Traumatology Center, São Paulo - SP, Brasil. Correspondence: Rua Borges Lagoa, 783, 5 andar, São Paulo, SP, Brazil. mcastur@yahoo.com 
lower limbs for more than three weeks were evaluated and treated with ESWT and physiotherapy.

Inclusion criteria were: (1) Skeletally mature patients aged 18 years or older; (2) Muscle injury in the lower limbs; (3) Muscle injury grade II or grade III diagnosed by ultrasound examination; (4) Muscle injury for more than three weeks; (5) Professional or amateur athletes. The skeletally immature patients; those who had any other associated injury in the lower limbs; those who have not accepted or signed the Free and Informed Consent form were not included in the study. Exclusion criteria were: patients who abandoned treatment or did not attend evaluations during the proposed period.

After being diagnosed with muscle injury by physical examination and imaging (ultrasound), patients who fulfilled all inclusion criteria were asked to participate in the proposed treatment.

After inclusion, all patients were required to follow the pre-determined protocol. In the physical evaluation the following data were recorded: location of the injury, presence of palpable gap, evaluation of muscle strength and pain with the aid of a visual analogue scale. (Figure 1) The visual analogue pain scale (VAS) presents numbers from zero to 10 in ascending order from left to right. Zero means no pain and 10 means the worst pain imaginable. Thus, the patient had to choose the number that best represents his/her pain at that time. Clinical evaluation occurred every two weeks until completing six weeks. In sonographic evaluation there was measurement and classification of the injury, presence of hematoma, fibrosis and other measurements. The imaging evaluation occurred every two weeks until completing six weeks. (Figure 2) In addition, the Tegner questionnaire was used for subjective assessment of the patient on his/her ability to perform sports activity. ${ }^{12}$

After six weeks, the patient was discharged from physiotherapy and authorized to return to previously performed sports activities. After two weeks a questionnaire about his return to sports practice was applied (at eight weeks of treatment).

\section{Extracorporeal Shock Wave Therapy (ESWT)}

Extracorporeal Shock Wave Therapy (ESWT) was applied the day after the initial evaluation. ESWT was performed through an Evotron-Switech device with energy flux density of 0.03-0.06 mJ/ $\mathrm{mm}^{2}$. Overall, 900 pulses are applied per injury at 240 pulses/ min frequency. There was no need of anesthetic procedure for conducting the treatment. A second ESWT session was held after 3 weeks, if necessary (persistent functional disability). (Figure 3)

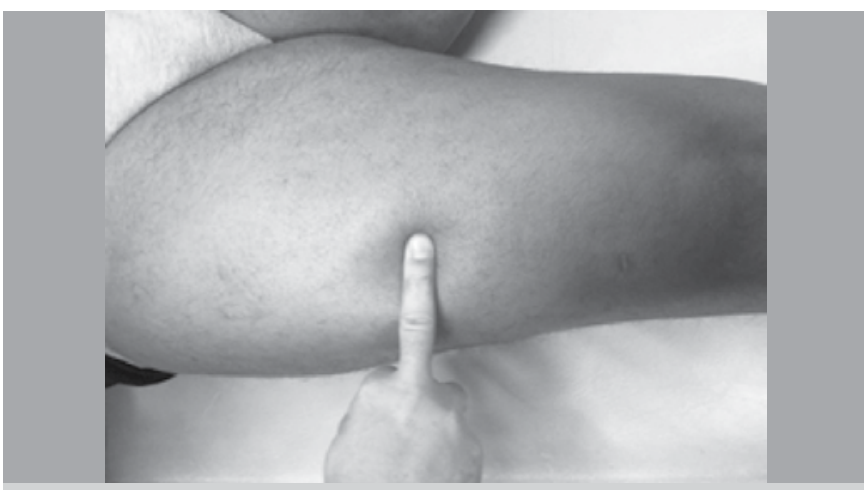

Figure 1. Presence of a gap detected at physical examination in the place where the patient reports pain, featuring a more severe quadriceps muscle injury.

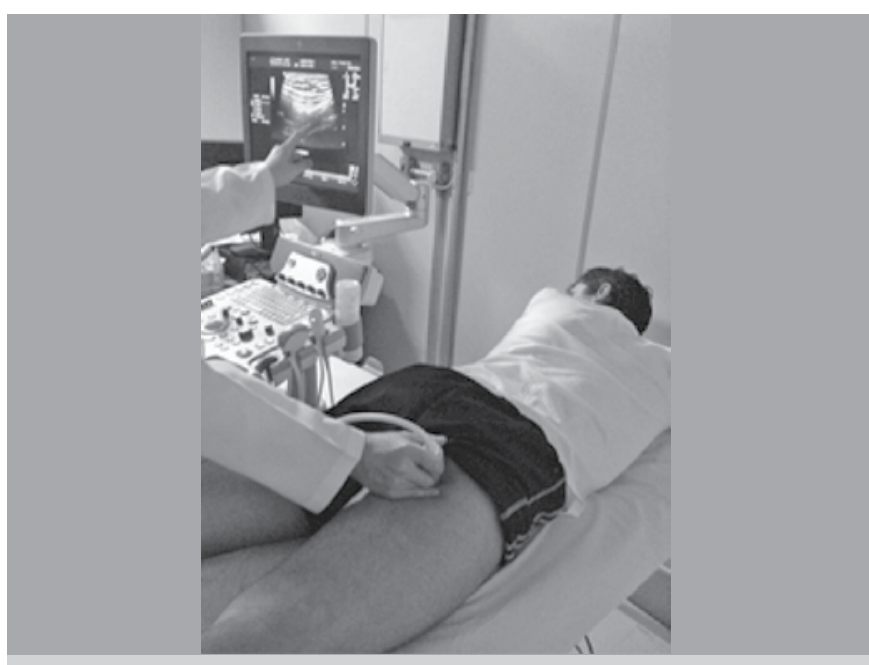

Figure 2. Physical examination performed at the same time of ultrasonography for evaluation and monitoring of muscle injury.

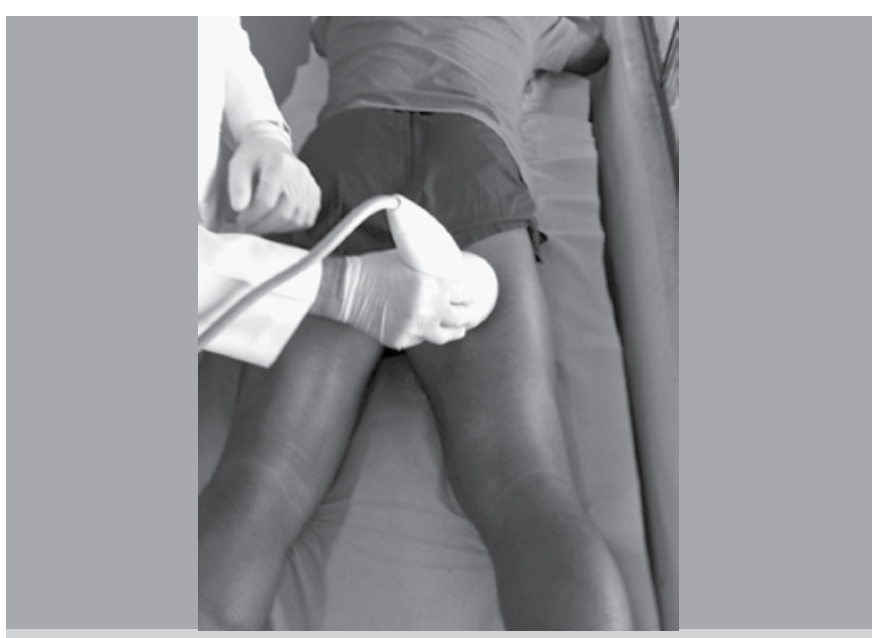

Figure 3. Twenty-six year old patient during the first ESWT session at posterior thigh muscle injury. The transducer is positioned directly over the injury site.

\section{Physiotherapy}

The patient was referred to physical therapy commencing the day after the first clinical evaluation, after the first ESWT session. The physical therapy protocol lasted four to six weeks. The physical therapy protocol consisted of three weekly sessions of isometric exercises with progressively increasing load, carried out on the edge of the patient's pain. In the second stage, started as soon as the movement performed in the first stage was painless, isotonic exercise was added. Finally, the third stage was based on isokinetic exercise until the injured limb muscle strength reached up to $90 \%$ of the contralateral healthy limb.

\section{RESULTS}

The mean age of the eight athletes treated was 29.1 years old (range, 19-51 years old). Three practiced athletics, two were football players, one jiu-jitsu fighter, one rugby player, and one pole dance athlete.

Seven athletes (87.5\%) had grade II injuries in the first assessment and only one had a grade III injury (12.5\%). Six athletes had 
damage to ischiotibial muscles (75\%) and two in the thigh adductor muscles (25\%).

Six athletes (75\%) presented the injury for the first time and two (25\%) reported not being the first injury episode at the site of the referred muscle damage.

The average injury time was 8.75 weeks (range, 4-16 weeks). In all cases the patients did not seek medical attention and tried to return to their sports activity as soon as they felt some improvement.

Five of the eight patients underwent two sessions of ESWT according to the previously defined protocol.

\section{Clinical Evaluation}

Palpable gap: Only patients with muscle injury grade III had palpable gap at the site of injury. After the end of treatment, it still showed visible sag of the skin at the site of injury, without interfering in the recovered function.

Muscle Strength: During the first evaluation, four patients had grade IV muscle strength on the affected limb, three patients had muscle strength grade $\mathrm{V}$, and one patient had muscle strength grade III. At the end of six weeks of treatment, all patients showed muscle strength grade $\mathrm{V}$.

Pain: The average points achieved before the start of treatment for VAS pain was 5.75 points (range, 3-8). After six weeks of treatment, this average decreased to 0.5 points (range, 0-2 points). Tegner: Before muscle damage, the mean score achieved by the athletes evaluated by Tegner scale was 8 points (range, 7-10). In the first consultation this average dropped to 3 points (range, 1-6). At the end of treatment we achieved an average of six points upon return to sports activities. (range, 5-7).

\section{Sonographic evaluation}

In the initial ultrasound assessment a collection of hematoma was found in one patient (4 weeks of injury). The presence of fibrotic tissue was described in all patients. The average size of the injury at the first examination was $4.2 \times 2.2 \times 2.1 \mathrm{~cm}$. At the end of treatment ( 6 weeks) the size of the region with echogenicity alteration was $2.2 \times 1.3 \times 1.1 \mathrm{~cm}$. (Figure 4)

\section{Return to sports activities}

The mean time to return to sports activities was 8.14 weeks after the first ESWT session. Only one athlete did not return to sports activities, however he claims willingly, even asymptomatic. A

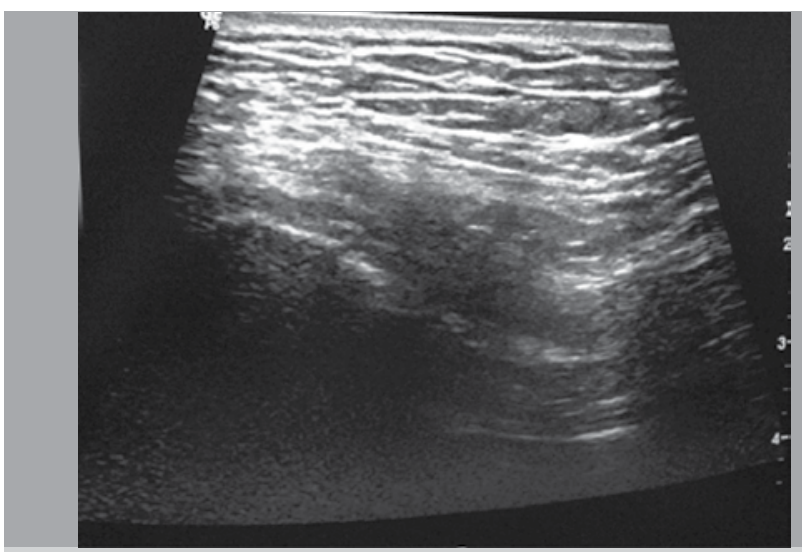

Figure 4. Image of a chronic muscle injury during treatment showing hypoechoic area in extensive region of the posterior muscles of the thigh.

Acta Ortop Bras. 2015;23(5):247-50 soccer player who returned to sports after 8 weeks from the last ESWT session, refers to train normally without pain on the run and kick. But he refers to a mild residual pain when required to start and crouch. This was one of two athletes that accounted previous injuries in the same location.

\section{DISCUSSION}

The muscle injury commonly occurs in professional and amateur athletes during their sports activities. Although the muscle is a structure with a good capacity for regeneration and return to its physiological function after treatment of an injury, often the desire to return to practice physical activity in a not long enough time for the complete recovery of its fibers makes the injury to remain and muscle function compromised. ${ }^{13}$

That is the most common way of a muscular injury to become chronic. There is no consensus in the literature regarding the time of injury for it to be considered chronic regarding muscle injury. However, more important than the time of injury are the changes in their histologic aspect that causes the muscle characteristics to make its treatment more complex. We considered as chronic injuries those which were not treated for at least three weeks from the date of injury and that show alterations in the ultrasound exam such as fibrosis and hematoma remains. Thus, we believe that fibrosis formed in the untreated injury is more difficult to be converted into a normal fiber than when treating an acute muscle injury, i.e., immediately after exercise. The treatment of muscle injury did not have any significant change over the last 40 years. Muscle injuries are classically treated with physiotherapy, rest from physical activities and pain medication. The results are very good when the injury is treated properly. Often the athlete does not pay the due attention to the injury, or returns to his/her activities prior to the time required for the recovery of the muscle fiber. The continued and intense use of the injured muscle fiber is what makes chronic an injury. In these cases, many times fibrosis and muscular scar formed are not amenable to cure only with physical therapy. In this interim, new therapies have emerged in order to improve and accelerate patient recovery: muscle positioning tape (Kinesio ${ }^{\circledR}$ Taping), a method designed from adhesive elastic bands applied over and around muscles, based on the cutaneous stimulating concept; Platelet rich plasma (PRP); therapeutic ultrasound (US); and hyperbaric therapy. ${ }^{14}$ However, few studies provide with enough background to use on a large scale.

ESWT therapy is also expected to produce an improvement in musculoskeletal healing and regeneration by a single acoustic wave impulse able to stimulate healing of soft tissue, mainly by inhibiting the function of afferent pain receptors and increases angiogenesis. It, thus, increases blood flow at the treatment site inducing a healing process mediated by the generated inflammatory process. ${ }^{10,11}$

Over the past 20 years ESWT has been widely employed in the treatment of enthesopathy. ${ }^{15}$ Clinical trials evaluated its effect in the treatment of plantar fasciitis, ${ }^{16}$ epicondylitis, ${ }^{17}$ patellar tendinitis, ${ }^{18}$ calcific and non-calcific rotator cuff tendinosis, ${ }^{19}$ and tendinopathy of Achilles tendon. ${ }^{20}$ The rate of success varies from $65 \%$ to $91 \%$ and complications are low and negligible. ${ }^{10}$ But little is known about its benefits applied to the treatment of muscle injury, especially when it was not handled properly, as the result of an earlier injury in the same location, or when the 
patient returned to sports practice before healing the injured fiber. We called these situations in our study as long-time injury, or chronic injury, which we define as the situations cited more than three weeks of the initial episode.

Although having described a case series, the improvement of patients with ESWT associated with physiotherapy was quite significant, with return to sports activities, improvement of symptoms and limitations imposed by the injury. The sonographic appearance of the lesion did not change so quickly, but there was a progressive evolution of their appearance. The visual analogue pain scale after treatment reached its minimum value in $75 \%$ of patients, muscle strength returned to its maximum value in all patients, and return to sports activities was possible in all those who so wished. Thus, although no other study mentions the use of this therapeutic technique for the treatment of muscle injuries, we believe it is a useful option for the relief of symptoms and return to sports practice in patients with this type of injury.
Failure to compare patients who received ESWT and physical therapy with a group undergoing conventional treatment was one of the limiting factors of the study. Comparing physiotherapy treatment alone with physical therapy associated with ESWT would be a better way to assess its real benefits. However, only with the documentation of a number of cases it was possible to assess the progressive evolution to clinical improvement of the patient. Furthermore, the sample could be expanded to a better significance of data. However, the difficulty of homogenization of the sample according with the type, location, degree and duration of the injuries hindered this option.

\section{CONCLUSION}

All patients with chronic injury of the muscles of the lower limbs treated with physical therapy and ESWT protocol showed improvement of pain and muscle strength after the treatment. Additionally, all patients were able to return to sports practice on previously performed physical activity.

\section{REFERENCES}

1. Ahmad CS, Redler LH, Ciccotti MG, Maffulli N, Longo UG, Bradley J. Evaluation and management of hamstring injuries. Am J Sports Med. 2013;41(12):2933-47.

2. Andersen TE, Engebretsen L, Bahr R. Rule violations as a cause of injuries in male norwegian professional football: are the referees doing their job? Am J Sports Med. 2004;32(Suppl 1):62S-8S.

3. Brophy RH, Wright RW, Powell JW, Matava MJ. Injuries to kickers in American football: the National Football League experience. Am J Sports Med. 2010;38(6):1166-73.

4. Ekstrand J, Hägglund M, Waldén M. Epidemiology of muscle injuries inprofessional football (soccer). Am J Sports Med. 2011;39(6):1226-32.

5. Borowski LA, Yard EE, Fields SK, Comstock RD. The epidemiology of US high school basketball injuries, 2005-2007. Am J Sports Med. 2008;36(12):2328-35.

6. Lopez V Jr, Galano GJ, Black CM, Gupta AT, James DE, Kelleher KM, et al. Profile of an American amateur rugby union sevens series. Am J Sports Med. 2012;40(1):179-84

7. Malliaropoulos N, Isinkaye T, Tsitas K, Maffulli N. Reinjury after acute posterior thigh muscle injuries in elite track and field athletes. Am J Sports Med. 2011;39(2):304-10.

8. Järvinen TA, Järvinen TL, Kääriäinen $M$, Kalimo H, Järvinen M. Muscle injuries: biology and treatment. Am J Sports Med. 2005;33(5):745-64.

9. Opar DA, Williams MD, Shield AJ. Hamstring strain injuries: factors that lead to injury and re-injury. Sports Med. 2012;42(3):209-26.

10. Wang CJ. Extracorporeal shockwave therapy in musculoskeletal disorders. J Orthop Surg Res. 2012;7:11
11. Wilson M, Stacy J. Shock wave therapy for Achilles tendinopathy. Curr Rev Musculoskelet Med. 2010;4(1):6-10.

12. Tegner $Y$, Lysholm J. Rating systems in the evaluation of knee ligament injuries. Clin Orthop Relat Res. 1985;(198):43-9.

13. Taylor DC, Dalton JD Jr, Seaber AV, Garrett WE Jr. Experimental muscle strain injury. Early functional and structural deficits and the increased risk for reinjury. Am J Sports Med. 1993;21(2):190-4.

14. Best TM, Loitz-Ramage B, Corr DT, Vanderby R. Hyperbaric oxygen in the treatment of acute muscle stretch injuries. Results in an animal Model. Am J Sports Med. 1998;26(3):367-72.

15. Sems A, Dimeff R, lannotti JP. Extracorporeal shock wave therapy in the treatment of chronic tendinopathies. J Am Acad Orthop Surg. 2006;14(4):195-204.

16. Rompe JD, Schoellner C, Nafe B. Evaluation of low-energy extracorporeal shock-wave application for treatment of chronic plantar fasciitis. J Bone Joint Surg Am. 2002;84(3):335-41.

17. Ko JY, Chen HS, Chen LM. Treatment of lateral epicondylitis of the elbow with shock waves. Clin Orthop Relat Res. 2001;(387):60-7.

18. Russo S, de Durante C, Gigliotti S, Galasso O. Shock wave management of footballer's tendinopathies. J Sports Traumatol Rel Res. 1999;21:84-8.

19. Huisstede BM, Gebremariam L, van der Sande R, Hay EM, Koes BW. Evidence for effectiveness of Extracorporal Shock-Wave Therapy (ESWT) to treat calcific and non-calcific rotator cuff tendinosis: a systematic review. Man Ther. 2011;16(5):419-33.

20. Astor F, Sansone V, De Marchi F. Extracorporeal shock wave therapy for tendinopathy of Achilles. In: 23rd Isokinetic congress on rehabilitation of sports muscle and tendon injuries, Turin, Italy, 2004 